\title{
Correlations of Eigenfunctions in Disordered Systems
}

\author{
Ya. M. Blanter ${ }^{a, b}$ and A. D. Mirlin ${ }^{a, c}$ \\ ${ }^{a}$ Institut für Theorie der Kondensierten Materie, Universität Karlsruhe, 76128 Karlsruhe, Germany \\ ${ }^{b}$ Department of Theoretical Physics, Moscow Institute for Steel and Alloys, Leninskii Pr. 4, 117936 Moscow, Russia \\ ${ }^{c}$ Petersburg Nuclear Physics Institute, 188350 Gatchina, St. Petersburg, Russia
}

(July 2, 2021)

Correlations of eigenfunctions, $\left\langle\left|\psi_{k}\left(r_{1}\right)\right|^{2}\left|\psi_{l}\left(r_{2}\right)\right|^{2}\right\rangle$, in a disordered system are investigated. We derive general formulae expressing these correlation functions in terms of the supermatrix sigma-model. In particular case of weak localization regime we find that the correlations of the same eigenfunction are proportional to $g^{-1}$ for large distances, while the correlations of two different eigenfunctions cross over from $g^{-1}$ behavior for $r_{1}=r_{2}$ to $g^{-2}$ one for $\left|r_{1}-r_{2}\right| \gg l$, with $g$ and $l$ being the dimensionless conductance and the mean free path, respectively.

PACS numbers: 05.45+b, 72.15.-v, 73.20.Dx

Statistics of eigenfunction fluctuations in disordered and chaotic systems have attracted a research interest recently. The fluctuations of eigenfunction amplitudes determine statistical properties of conductance peaks and level width in quantum dots in the Coulomb blockade regime [1 5], and can be directly measured in the microwave cavity experiments [6]7]. On the theoretical side, the recent progress is based on application of the supersymmetry method to the problem of eigenfunction statistics [8,2]. It was found that the distribution of eigenfunction amplitudes is in the leading (zero-mode) approximation correctly described by formulae of the random matrix theory (RMT). Deviations from the RMT predictions were studied in Refs. 8 10]. Correlations of amplitudes of an eigenfunction in two different spatial points were considered in Ref. [11] on the level of zero-mode approximation; the latter was shown [12] to be equivalent to the RMT-like assumption of the Gaussian fluctuations of wavefunctions.

All results mentioned above concern fluctuations of the same eigenfunction. In the present Letter we study for the first time correlations of amplitudes of two different eigenfunctions. We derive general expressions in terms of the supermatrix sigma model, valid for arbitrary diffusive (or classically chaotic) system, and then apply them to the weak localization regime.

In order to evaluate the correlations of the wavefunctions we use the technique similar to that of Ref. [13]. Namely, we consider a quantity

$$
\begin{aligned}
& A\left(\boldsymbol{r}_{1}, \boldsymbol{r}_{2}, \omega\right) \\
& =\left\langle\sum_{k, l}\left|\psi_{k}\left(\boldsymbol{r}_{1}\right) \psi_{l}\left(\boldsymbol{r}_{2}\right)\right|^{2} \delta\left(\epsilon-\epsilon_{k}\right) \delta\left(\epsilon+\omega-\epsilon_{l}\right)\right\rangle-
\end{aligned}
$$

$$
\left\langle\sum_{k}\left|\psi_{k}\left(\boldsymbol{r}_{1}\right)\right|^{2} \delta\left(\epsilon-\epsilon_{k}\right)\right\rangle\left\langle\sum_{l}\left|\psi_{l}\left(\boldsymbol{r}_{2}\right)\right|^{2} \delta\left(\epsilon+\omega-\epsilon_{l}\right)\right\rangle .
$$

Here the angular brackets denote the impurity average. We have introduced the eigenstates $\psi_{k}(\boldsymbol{r})$ and eigenvalues $\epsilon_{k}$ of the Hamiltonian $\hat{H}=\hat{H}_{0}+U(\boldsymbol{r})$ in a particular disorder configuration $U(\mathbf{r}), H_{0}$ being the Hamiltonian of the free particle. Further we denote

$$
\begin{aligned}
\alpha\left(\boldsymbol{r}_{1}, \boldsymbol{r}_{2}, \epsilon_{k}\right) & =\left\langle\left|\psi_{k}\left(\boldsymbol{r}_{1}\right) \psi_{k}\left(\boldsymbol{r}_{2}\right)\right|^{2}\right\rangle \\
\beta\left(\boldsymbol{r}_{1}, \boldsymbol{r}_{2}, \epsilon_{k}, \epsilon_{l}\right) & =\left\langle\left|\psi_{k}\left(\boldsymbol{r}_{1}\right) \psi_{l}\left(\boldsymbol{r}_{2}\right)\right|^{2}\right\rangle, \quad k \neq l,
\end{aligned}
$$

where the averaging is carried over all eigenstates $\psi_{k}, \psi_{l}$ with given values of the energies $\epsilon_{k}, \epsilon_{l}$. Since we are interested in a relatively narrow window in the spectrum, $\omega \ll \epsilon_{F}$, these quantities are translationally invariant:

$$
\begin{aligned}
\alpha\left(\boldsymbol{r}_{1}, \boldsymbol{r}_{2}, \epsilon_{k}\right) & =\alpha\left(\boldsymbol{r}_{1}, \boldsymbol{r}_{2}\right), \\
\beta\left(\boldsymbol{r}_{1}, \boldsymbol{r}_{2}, \epsilon_{k}, \epsilon_{l}\right) & =\beta\left(\boldsymbol{r}_{1}, \boldsymbol{r}_{2}, \epsilon_{k}-\epsilon_{l}\right) .
\end{aligned}
$$

Due to the presence of delta-functions in Eq. (1), we can rewrite the latter exactly as

$$
\begin{aligned}
A\left(\boldsymbol{r}_{1}, \boldsymbol{r}_{2}, \omega\right) & =\alpha\left(\boldsymbol{r}_{1}, \boldsymbol{r}_{2}\right) \Delta^{-1} \delta(\omega) \\
& +\beta\left(\boldsymbol{r}_{1}, \boldsymbol{r}_{2}, \omega\right) \Delta^{-2} R(\omega),
\end{aligned}
$$

where $\Delta$ is the mean level spacing, $\Delta=(\nu V)^{-1}$, with $V$ and $\nu$ being the system volume and the density of states, respectively. Here we have introduced the twolevel correlation function,

$$
R(\omega)=\Delta^{2}\left\langle\sum_{k \neq l} \delta\left(\epsilon-\epsilon_{k}\right) \delta\left(\epsilon+\omega-\epsilon_{l}\right)\right\rangle .
$$

We would like to stress that all transformations up to now are completely rigorous. In particular, we do not assume any decoupling of eigenfunction and eigenvalue correlations.

On the other hand, the quantity (11) can be written in terms of the Green's functions in the coordinatefrequency representation,

$$
\begin{aligned}
& A\left(\boldsymbol{r}_{1}, \boldsymbol{r}_{2}, \omega\right)=\left(2 \pi^{2}\right)^{-1} \\
\times & \operatorname{Re}\left\{\left\langle G^{R}\left(\boldsymbol{r}_{1}, \boldsymbol{r}_{1}, \epsilon\right) G^{A}\left(\boldsymbol{r}_{2}, \boldsymbol{r}_{2}, \epsilon+\omega\right)\right\rangle\right. \\
- & \left.\left\langle G^{R}\left(\boldsymbol{r}_{1}, \boldsymbol{r}_{1}, \epsilon\right)\right\rangle\left\langle G^{A}\left(\boldsymbol{r}_{2}, \boldsymbol{r}_{2}, \epsilon+\omega\right)\right\rangle\right\} .
\end{aligned}
$$

The expression (6) can be directly calculated with the use of the supersymmetry technique $[14-16$. We concentrate in the sequel on the case of broken time-reversal 
symmetry (unitary ensemble); generalization to the other ensembles is straightforward. After the standard manipulations we get

$$
\begin{aligned}
& A\left(\boldsymbol{r}_{1}, \boldsymbol{r}_{2}, \omega\right)=-\left(2 \pi^{2}\right)^{-1} \operatorname{Re}\left\{\left\langleg_{b b}^{11}\left(\boldsymbol{r}_{1}, \boldsymbol{r}_{1}\right) g_{b b}^{22}\left(\boldsymbol{r}_{2}, \boldsymbol{r}_{2}\right)\right.\right. \\
& \left.+g_{b b}^{12}\left(\boldsymbol{r}_{1}, \boldsymbol{r}_{2}\right) g_{b b}^{21}\left(\boldsymbol{r}_{2}, \boldsymbol{r}_{1}\right)\right\rangle_{F}-\left\langle g_{b b}^{11}\left(\boldsymbol{r}_{1}, \boldsymbol{r}_{1}\right)\right\rangle_{F} \\
& \left.\times\left\langle g_{b b}^{22}\left(\boldsymbol{r}_{2}, \boldsymbol{r}_{2}\right)\right\rangle_{F}\right\} .
\end{aligned}
$$

Here $\langle\ldots\rangle_{F}$ denotes the averaging with the action of the supermatrix sigma-model $F[Q]$ :

$$
\begin{aligned}
& \langle\ldots\rangle_{F}=\int D Q(\ldots) \exp (-F[Q]), \\
& F[Q]=-\frac{\pi \nu}{4} \int d \boldsymbol{r} \operatorname{Str}\left[D(\nabla Q)^{2}+2 i(\omega+i 0) \Lambda Q\right],
\end{aligned}
$$

where $D$ is the diffusion coefficient, $Q=T^{-1} \Lambda T$ is a $4 \times 4$ supermatrix, $\Lambda=\operatorname{diag}(1,1,-1,-1)$, and $T$ belongs to the supercoset space $U(1,1 \mid 2) / U(1 \mid 1) \times U(1 \mid 1)$. The symbol Str denotes the supertrace defined as $\operatorname{Str} B=$ $B_{b b}^{11}-B_{f f}^{11}+B_{b b}^{22}-B_{f f}^{22}$. The upper matrix indices correspond to the retarded-advanced decomposition, while the lower indices denote the boson-fermion one. The Green's function $g$ in Eq. (7) is the solution to the matrix equation:

$$
\begin{aligned}
& {\left[-i\left(\epsilon+\frac{\omega}{2}-\hat{H}_{0}\right)-\frac{i}{2}(\omega+i 0) \Lambda+Q / 2 \tau\right] g\left(\boldsymbol{r}_{1}, \boldsymbol{r}_{2}\right)} \\
& =\delta\left(\boldsymbol{r}_{1}-\boldsymbol{r}_{2}\right) .
\end{aligned}
$$

Expressing these functions through the matrices $Q$ and taking into account Eq. (4), we arrive at the following equation valid in arbitrary diffusive system:

$$
\begin{aligned}
& 2 \pi^{2}\left[\frac{\alpha\left(\boldsymbol{r}_{1}, \boldsymbol{r}_{2}\right)}{\Delta} \delta(\omega)+\frac{\beta\left(\boldsymbol{r}_{1}, \boldsymbol{r}_{2}, \omega\right)}{\Delta^{2}} R(\omega)\right] \\
& =-(\pi \nu)^{2} \operatorname{Re}\left\langle Q_{b b}^{11}\left(\boldsymbol{r}_{1}\right) Q_{b b}^{22}\left(\boldsymbol{r}_{2}\right)\right\rangle_{F} \\
& -\left[\operatorname{Im} G^{R}\left(\boldsymbol{r}_{1}-\boldsymbol{r}_{2}\right)\right]^{2} \operatorname{Re}\left\langle Q_{b b}^{12}\left(\boldsymbol{r}_{1}\right) Q_{b b}^{21}\left(\boldsymbol{r}_{1}\right)\right\rangle_{F}-(\pi \nu)^{2}
\end{aligned}
$$

with $G^{R}$ being the impurity averaged retarded Green's function. In particular, in the case of $2 \mathrm{D}$ and $3 \mathrm{D}$ system, $G^{R}$ is given by

$$
G^{R}(\boldsymbol{r})= \begin{cases}-i \nu \int_{-\pi / 2}^{\pi / 2} d \theta \exp \left[\left(i p_{F} r-r / 2 l\right) \cos \theta\right], & 2 D \\ -\pi \nu\left(p_{F} r\right)^{-1} \exp \left[i p_{F} r-r / 2 l\right], & 3 D\end{cases}
$$

where $l$ is the mean free path. The key point making the further progress possible is that the term containing the single eigenfunction correlations in lhs of Eq.(10) is proportional to $\delta(\omega)$, whereas the one depending on the correlations of two different eigenfunctions is regular at $\omega=0$. Thus, separation of the expression in the rhs of Eq. (10) into the singular (proportional to $\delta(\omega)$ ) and regular parts allows one to obtain the quantities $\alpha\left(\boldsymbol{r}_{1}, \boldsymbol{r}_{2}\right)$ and $\beta\left(\boldsymbol{r}_{1}, \boldsymbol{r}_{2}, \omega\right)$.
Now we turn to the case of a metallic system in the weak localization regime. The corresponding small parameter is given by Eq. (19) below. For further purposes, we introduce the functions

$$
\begin{aligned}
f_{1}\left(\boldsymbol{r}_{1}, \boldsymbol{r}_{2}\right) & =\Pi^{2}\left(\boldsymbol{r}_{1}, \boldsymbol{r}_{2}\right), \\
f_{2}\left(\boldsymbol{r}_{1}, \boldsymbol{r}_{2}\right) & =(2 V)^{-1} \int d \boldsymbol{r}\left[\Pi^{2}\left(\boldsymbol{r}, \boldsymbol{r}_{1}\right)+\Pi^{2}\left(\boldsymbol{r}, \boldsymbol{r}_{2}\right)\right], \\
f_{3} & =V^{-2} \int d \boldsymbol{r} d \boldsymbol{r}^{\prime} \Pi^{2}\left(\boldsymbol{r}, \boldsymbol{r}^{\prime}\right) \\
f_{4}\left(\boldsymbol{r}_{1}, \boldsymbol{r}_{2}\right) & =V^{-1} \int d \boldsymbol{r} \Pi\left(\boldsymbol{r}, \boldsymbol{r}_{1}\right) \Pi\left(\boldsymbol{r}, \boldsymbol{r}_{2}\right) .
\end{aligned}
$$

Here the diffusion propagator $\Pi$ is the solution to the diffusion equation

$$
-D \nabla^{2} \Pi\left(\boldsymbol{r}_{1}, \boldsymbol{r}_{2}\right)=(\pi \nu)^{-1} \delta\left(\boldsymbol{r}_{1}-\boldsymbol{r}_{2}\right)
$$

with appropriate boundary conditions. We obtain:

$$
\Pi\left(\boldsymbol{r}_{1}, \boldsymbol{r}_{2}\right)=(\pi \nu V)^{-1} \sum_{\boldsymbol{q}}\left(D q^{2}\right)^{-1} \phi_{\boldsymbol{q}}\left(\boldsymbol{r}_{1}\right) \phi_{\boldsymbol{q}}\left(\boldsymbol{r}_{2}\right),
$$

with $\phi_{\boldsymbol{q}}$ being the eigenfunction of the diffusion operator corresponding to the eigenvalue $D q^{2}, \boldsymbol{q} \neq 0$. The level correlation function has the form 17.

$$
R(\omega)=1-s^{-2} \sin ^{2} s+f_{3} \sin ^{2} s+O\left(g^{-3}\right),
$$

where a dimensionless parameter $s=\pi \omega / \Delta$ is introduced. The first two terms in Eq. (14) are given by RMT, while the third one is the correction of order $g^{-2}$ due to the diffusion modes. Here $g=2 \pi E_{c} / \Delta$ is the dimensionless conductance, with $E_{c}$ being the Thouless energy.

The sigma-model correlation functions $\left\langle Q_{b b}^{11}\left(\boldsymbol{r}_{1}\right) Q_{b b}^{22}\left(\boldsymbol{r}_{2}\right)\right\rangle_{F}$ and $\left\langle Q_{b b}^{12}\left(\boldsymbol{r}_{1}\right) Q_{b b}^{21}\left(\boldsymbol{r}_{2}\right)\right\rangle_{F}$ can be calculated for relatively low frequencies $\omega \ll E_{c}$ with the use of a general method developed in Refs. [17,9] which allows one to take into account spatial variations of the field $Q$. The results are obtained in form of expansions in $g^{-1}$. First, we restrict ourselves to the terms of order $g^{-1}$. Then, the result for the first correlator reads as

$$
\begin{aligned}
& \left\langle Q_{b b}^{11}\left(\boldsymbol{r}_{1}\right) Q_{b b}^{22}\left(\boldsymbol{r}_{2}\right)\right\rangle_{F} \\
& \quad=-1-2 i \frac{\exp (i s) \sin s}{(s+i 0)^{2}}-2 i \frac{1}{s+i 0} \Pi\left(\boldsymbol{r}_{1}, \boldsymbol{r}_{2}\right) .
\end{aligned}
$$

The first two terms in Eq. (15) represent the result of the so-called zero-mode approximation [14], which takes into account only the spatially constant configurations of the field $Q(\boldsymbol{r})$, so that the functional integral over $D Q(\boldsymbol{r})$ is reduced to an integral over a single matrix $Q$. The last term is the correction of order $g^{-1}$. An analogous calculation for the second correlator yields [13]:

$$
\begin{gathered}
\left\langle Q_{b b}^{12}\left(\boldsymbol{r}_{1}\right) Q_{b b}^{21}\left(\boldsymbol{r}_{2}\right)\right\rangle_{F}=-2\left\{\frac{i}{s+i 0}\right. \\
\left.+\left[1+i \frac{\exp (i s) \sin s}{(s+i 0)^{2}}\right] \Pi\left(\boldsymbol{r}_{1}, \boldsymbol{r}_{2}\right)\right\} .
\end{gathered}
$$


Now, separating regular and singular parts in rhs of Eq. (10), we obtain the following result for the autocorrelations of the same eigenfunction:

$$
\begin{aligned}
& V^{2}\left\langle\left|\psi_{k}\left(\boldsymbol{r}_{1}\right) \psi_{k}\left(\boldsymbol{r}_{2}\right)\right|^{2}\right\rangle-1 \\
& =k_{d}(r)\left[1+\Pi\left(\boldsymbol{r}_{1}, \boldsymbol{r}_{1}\right)\right]+\Pi\left(\boldsymbol{r}_{1}, \boldsymbol{r}_{2}\right),
\end{aligned}
$$

and for the correlation of amplitudes of two different eigenfunctions

$$
V^{2}\left\langle\left|\psi_{k}\left(\boldsymbol{r}_{1}\right) \psi_{l}\left(\boldsymbol{r}_{2}\right)\right|^{2}\right\rangle-1=k_{d}(r) \Pi\left(\boldsymbol{r}_{1}, \boldsymbol{r}_{1}\right), \quad k \neq l
$$

Here $r=\left|\boldsymbol{r}_{1}-\boldsymbol{r}_{2}\right|$, and the function $k_{d}(r)$ is defined as

$$
\begin{aligned}
& k_{d}(r)=(\pi \nu)^{-2}\left[\operatorname{Im} G^{R}(\boldsymbol{r})\right]^{2}= \\
& =\exp (-r / l)\left\{\begin{array}{lll}
1, & 2 D, & p_{F} r \ll 1 \\
8\left(\pi p_{F} r\right)^{-1} \cos ^{2} p_{F} r, & 2 D, & p_{F} r \gg 1 \\
\left(p_{F} r\right)^{-2} \sin ^{2} p_{F} r, & 3 D
\end{array}\right.
\end{aligned}
$$

Note that the result (17) for $\boldsymbol{r}_{1}=\boldsymbol{r}_{2}$ is the inverse participation ratio previously obtained in Ref. [9], while that for arbitrary spatial separation was found in the zero-mode approximation $(g=\infty)$ in Ref. [11].

Eq. (18) shows that the correlations between different eigenfunctions are relatively small in the weak disorder regime. Indeed, they are proportional to the small parameter $\Pi(\boldsymbol{r}, \boldsymbol{r})$, which is equal in the cases of $2 \mathrm{D}$ and $3 \mathrm{D}$ geometry to ( $L$ is the size of the system)

$$
\Pi(\boldsymbol{r}, \boldsymbol{r})= \begin{cases}(\pi g)^{-1} \ln L / l, & 2 D \\ \sim g^{-1} L / l, & 3 D\end{cases}
$$

In particular, for $\boldsymbol{r}_{1}=\boldsymbol{r}_{2}$ we have

$$
\left.\left.V^{2}\langle| \psi_{k}(\boldsymbol{r}) \psi_{l} \boldsymbol{r}\right)\left.\right|^{2}\right\rangle-1=\delta_{k l}+\left(1+\delta_{k l}\right) \Pi(\boldsymbol{r}, \boldsymbol{r}) .
$$

The correlations are enhanced by disorder; when the system approaches the mobility edge in $3 \mathrm{D}$ or the strong localization regime in $2 \mathrm{D}$, the relative magnitude of correlations, $\Pi(\boldsymbol{r}, \boldsymbol{r})$ becomes the quantity of order of unity.

An inspection of Eqs. (17), (18) shows that while the correlations of amplitude of the same wavefunction survives for the large separation between the points, $r \gg l$, and is proportional to $g^{-1}$, the correlations of two different wavefunctions decay exponentially for the distances larger than the mean free path $l$. This is, however, an artifact of the $g^{-1}$ approximation, and the investigation of the corresponding tails requires the extension of the above calculation to the terms proportional to $g^{-2}$. We find that the correlator $\left\langle Q_{b b}^{11} Q_{b b}^{22}\right\rangle_{F}$ gets the following correction:

$$
\begin{aligned}
\delta\left\langle Q_{b b}^{11} Q_{b b}^{22}\right\rangle_{F}=-f_{1}+2 f_{4}+\exp (2 i s) f_{3} \\
-2 i \frac{\exp (2 i s)}{s+i 0}\left(f_{2}-f_{3}\right) \\
\quad-\frac{\exp (2 i s)-1}{2(s+i 0)^{2}}\left(f_{1}-4 f_{2}+3 f_{3}-4 f_{4}\right) .
\end{aligned}
$$

Consequently, we obtain the following results for the correlations of different eigenfunctions at $r>l$ :

$$
\begin{gathered}
V^{2}\left\langle\left|\psi_{k}\left(\boldsymbol{r}_{1}\right) \psi_{l}\left(\boldsymbol{r}_{2}\right)\right|^{2}\right\rangle-1=\frac{1}{2}\left(1-\frac{\sin ^{2} s}{s^{2}}\right)^{-1} \\
\times\left[f_{1}-f_{3}-2 f_{4}-\frac{2 \sin (2 s)}{s}\left(f_{2}-f_{3}\right)\right. \\
\left.\quad-\frac{\sin ^{2} s}{s^{2}}\left(f_{1}-4 f_{2}+3 f_{3}-2 f_{4}\right)\right], \quad k \neq l
\end{gathered}
$$

As it should be expected, the integrals over the coordinate of this correlation function are equal to zero. This property is just the normalization condition and should hold in arbitrary order of expansion in $g^{-1}$. Note also that the correlation of different eigenfunctions becomes now frequency dependent in a non-trivial way, even in the regime $\omega \ll E_{c}$ considered.

The quantities $f_{2}, f_{3}$, and $f_{4}$ are proportional to $g^{-2}$, with some numerical prefactors [17]. On the other hand, $f_{1}$ depends essentially on the distance $r=\left|\boldsymbol{r}_{1}-\boldsymbol{r}_{2}\right|$. In particular, for $l \ll r \ll L$ we find

$$
f_{1}\left(\boldsymbol{r}_{1}, \boldsymbol{r}_{2}\right)=\Pi^{2}\left(\boldsymbol{r}_{1}, \boldsymbol{r}_{2}\right) \approx\left\{\begin{array}{l}
\frac{1}{(\pi g)^{2}} \ln ^{2} \frac{L}{r} \\
\frac{1}{\left(4 \pi^{2} \nu D r\right)^{2}},
\end{array}\right.
$$

Thus, for $l<r \ll L$, the contributions proportional to $f_{1}$ dominate in Eq.(22), and we get

$$
V^{2}\left\langle\left|\psi_{k}\left(\boldsymbol{r}_{1}\right) \psi_{l}\left(\boldsymbol{r}_{2}\right)\right|^{2}\right\rangle-1=\frac{1}{2} \Pi^{2}\left(\boldsymbol{r}_{1}, \boldsymbol{r}_{2}\right), \quad k \neq l .
$$

Another correlation function, generally used for the calculation of the linear response of the system,

$$
\gamma\left(\boldsymbol{r}_{1}, \boldsymbol{r}_{2}, \omega\right)=\left\langle\psi_{k}^{*}\left(\boldsymbol{r}_{1}\right) \psi_{l}\left(\boldsymbol{r}_{1}\right) \psi_{k}\left(\boldsymbol{r}_{2}\right) \psi_{l}^{*}\left(\boldsymbol{r}_{2}\right)\right\rangle, \quad k \neq l,
$$

can be calculated in a similar way (cf. 13). Starting from the quantity

$$
\begin{aligned}
& B\left(\boldsymbol{r}_{1}, \boldsymbol{r}_{2}, \omega\right)= \\
& \left\langle\sum_{k, l} \psi_{k}^{*}\left(\boldsymbol{r}_{1}\right) \psi_{l}\left(\boldsymbol{r}_{1}\right) \psi_{k}\left(\boldsymbol{r}_{2}\right) \psi_{l}^{*}\left(\boldsymbol{r}_{2}\right) \delta\left(\epsilon-\epsilon_{k}\right) \delta\left(\epsilon+\omega-\epsilon_{l}\right)\right\rangle \\
& \quad-\left\langle\sum_{k} \psi_{k}^{*}\left(\boldsymbol{r}_{1}\right) \psi_{k}\left(\boldsymbol{r}_{2}\right) \delta\left(\epsilon-\epsilon_{k}\right)\right\rangle \\
& \quad \times\left\langle\sum_{l} \psi_{l}\left(\boldsymbol{r}_{1}\right) \psi_{l}^{*}\left(\boldsymbol{r}_{2}\right) \delta\left(\epsilon+\omega-\epsilon_{l}\right)\right\rangle,
\end{aligned}
$$

and repeating the derivation that led us to Eq. (10), we get another identity:

$$
\begin{aligned}
& 2 \pi^{2}\left[\frac{\alpha\left(\boldsymbol{r}_{1}, \boldsymbol{r}_{2}\right)}{\Delta} \delta(\omega)+\frac{\gamma\left(\boldsymbol{r}_{1}, \boldsymbol{r}_{2}, \omega\right)}{\Delta^{2}} R(\omega)\right] \\
& =-(\pi \nu)^{2} \operatorname{Re}\left\{\left\langle Q_{b b}^{12}\left(\boldsymbol{r}_{1}\right) Q_{b b}^{21}\left(\boldsymbol{r}_{2}\right)\right\rangle_{F}\right. \\
& \left.-k_{d}(r)\left\langle Q_{b b}^{11}\left(\boldsymbol{r}_{1}\right) Q_{b b}^{22}\left(\boldsymbol{r}_{1}\right)\right\rangle_{F}-k_{d}(r)\right\}
\end{aligned}
$$


Taking into account Eqs. (15) and (16), and separating the rhs into the regular and singular parts, we recover Eq. (17) and obtain

$$
\begin{aligned}
& V^{2}\left\langle\psi_{k}^{*}\left(\boldsymbol{r}_{1}\right) \psi_{l}\left(\boldsymbol{r}_{1}\right) \psi_{k}\left(\boldsymbol{r}_{2}\right) \psi_{l}^{*}\left(\boldsymbol{r}_{2}\right)\right\rangle \\
& =k_{d}(r)+\Pi\left(\boldsymbol{r}_{1}, \boldsymbol{r}_{2}\right), \quad k \neq l .
\end{aligned}
$$

As was mentioned, the above derivation is valid for $\omega \ll E_{c}$. In order to obtain the results in the range $\omega \gtrsim E_{c}$ one can calculate the sigma-model correlation functions entering Eqs. (10), (25) by means of the perturbation theory [18]. We find then for $k \neq l$

$$
\begin{aligned}
& V^{2}\left\langle\left|\psi_{k}\left(\boldsymbol{r}_{1}\right) \psi_{l}\left(\boldsymbol{r}_{2}\right)\right|^{2}\right\rangle=1+\operatorname{Re}\left\{k_{d}(r) \Pi_{\omega}\left(\boldsymbol{r}_{1}, \boldsymbol{r}_{2}\right)\right. \\
& \left.\quad+\frac{1}{2}\left[\Pi_{\omega}^{2}\left(\boldsymbol{r}_{1}, \boldsymbol{r}_{2}\right)-\frac{1}{V^{2}} \int d \boldsymbol{r} d \boldsymbol{r}^{\prime} \Pi_{\omega}^{2}\left(\boldsymbol{r}, \boldsymbol{r}^{\prime}\right)\right]\right\}, \quad(27) \\
& V^{2}\left\langle\psi_{k}^{*}\left(\boldsymbol{r}_{1}\right) \psi_{l}\left(\boldsymbol{r}_{1}\right) \psi_{k}\left(\boldsymbol{r}_{2}\right) \psi_{l}^{*}\left(\boldsymbol{r}_{2}\right)\right\rangle=k_{d}(r)+\operatorname{Re} \Pi_{\omega}\left(\boldsymbol{r}_{1}, \boldsymbol{r}_{2}\right),
\end{aligned}
$$

where $\Pi_{\omega}\left(\boldsymbol{r}_{1}, \boldsymbol{r}_{2}\right)$ is the finite-frequency diffusion propagator

$$
\Pi_{\omega}\left(\boldsymbol{r}_{1}, \boldsymbol{r}_{2}\right)=(\pi \nu V)^{-1} \sum_{\boldsymbol{q}} \frac{\phi_{q}\left(\boldsymbol{r}_{1}\right) \phi_{q}\left(\boldsymbol{r}_{2}\right)}{D q^{2}-i \omega},
$$

and the summation in Eq. (28) now includes $\boldsymbol{q}=0$.

The above derivation was performed for the case of a disordered sample. However, one can repeat it for a classically chaotic ballistic system, using the recent derivation of the supersymmetric sigma-model for this case [19]. In the end one gets the analogous results, but with the diffusion operator being replaced by the PerronFrobenius one for the given chaotic system. To analyze the results quantitatively in this case, one needs then an information about the eigenfunctions and eigenvalues of the Perron-Frobenius operator. This question deserves further investigation and goes beyond the scope of this Letter.

Finally, we discuss the relation between our results and the recent experiment [5], where strong correlations in amplitudes of neighboring conductance peaks of a quantum dot were observed. Within the one-electron picture, this seems to contradict our result (18) of weakness of correlations of different eigenfunctions. In principle, one could imagine that the dot was far from the universal (RMT) regime, so that the parameter $\Pi(\boldsymbol{r}, \boldsymbol{r})$ determining the magnitude of correlations was not small. This would be however in contradiction with the fact that the total distribution of peak heights in Ref. [5] was well described by the RMT formulae [1], since the corrections to the distribution of $\left|\psi^{2}(\boldsymbol{r})\right|$ (and consequently to that of peak heights) are proportional to the same parameter $\Pi(\boldsymbol{r}, \boldsymbol{r})[9]$. Another possibility is that the electronelectron interaction effects lead to some modification of eigenfunction correlations. This problem remains to be studied in future.

We are grateful to Charlie Marcus for a discussion which stimulated this research. The work was supported by the Alexander von Humboldt Foundation
(Y. M. B.) and SFB195 der Deutschen Forschungsgemeinschaft (A. D. M.).

[1] R. A. Jalabert, A. D. Stone, and Y. Alhassid, Phys. Rev. Lett. 68, 3468 (1992).

[2] V. N. Prigodin, K. B. Efetov, and S. Iida, Phys. Rev. Lett. 71, 1230 (1993).

[3] E. R. Mucciolo, V. N. Prigodin, and B. L. Altshuler, Phys. Rev. B 51, 1714 (1995).

[4] A. M. Chang, H. U. Baranger, L. N. Pfeiffer, K. W. West, and T. Y. Chang, Phys. Rev. Lett. 76, 1695 (1996).

[5] J. A. Folk, S. R. Patel, S. F. Godijn, A. G. Huibers, S. M. Cronenwett, C. M. Marcus, K. Campman, and A. C. Gossard, Phys. Rev. Lett. 76, 1699 (1996).

[6] H. J. Stöckmann and J. Stein, Phys. Rev. Lett. 64, 2215 (1990); J. Stein and H. J. Stöckmann, Phys. Rev. Lett. 68, 2867 (1992).

[7] S. Sridhar, Phys. Rev. Lett. 67, 785 (1991); A. Kudrolli, V. Kidambi, and S. Sridhar, Phys. Rev. Lett. 75, 822 (1995).

[8] A. D. Mirlin and Y. V. Fyodorov, J. Phys. A 26, L551 (1993).

[9] Y. V. Fyodorov and A. D. Mirlin, Pis'ma Zh. Eksp. Teor. Fiz. 60, 779 (1994) [JETP Lett. 60, 790 (1994)]; Phys. Rev. B 51, 13403 (1995).

[10] V.I.Fal'ko, K.B.Efetov, Phys. Rev. B 52, 17413 (1995); A.D.Mirlin, preprint cond-mat/9512095.

[11] V. N. Prigodin, Phys. Rev. Lett. 74, 1566 (1995); V. N. Prigodin, N. Taniguchi, A. Kudrolli, V. Kidambi, and S. Sridhar, Phys. Rev. Lett. 75, 2392 (1995).

[12] M. Srednicki, preprint cond-mat/9512115: M. Srednicki and F. Stiernelof, preprint chao-dyn/9603012.

[13] Ya. M. Blanter and A. D. Mirlin, Phys. Rev. B 53, 12601 (1996).

[14] K. B. Efetov, Adv. Phys. 32, 53 (1983).

[15] J. J. M. Verbaarschot, H. A. Weidenmüller, and M. R. Zirnbauer, Phys. Repts. 129, 367 (1985).

[16] M. R. Zirnbauer, Nucl. Phys. B265, 375 (1986).

[17] V. E. Kravtsov and A. D. Mirlin, Pis'ma Zh. Eksp. Teor. Fiz. 60, 645 (1994) [JETP Lett. 60, 656 (1994)].

[18] B. L. Altshuler and B. I. Shklovskii, Zh. Eksp. Theor. Fiz. 91, 220 (1986) [Sov. Phys. JETP 64, 127 (1986)].

[19] O. Agam, B. L. Altshuler, and A. V. Andreev, Phys. Rev. Lett. 75, 4389 (1995); A. V. Andreev, O. Agam, B. D. Simons, and B. L. Altshuler, Phys. Rev. Lett. 76, 3947 (1996); preprint cond-mat/9605204; B. A. Muzykantskii and D. E. Khmelnitskii, Pis'ma Zh. Eksp. Teor. Fiz. 62, 68 (1995) [JETP Lett. 62, 76 (1995)]. 